\title{
Antiarrhythmic drugs for out-of-hospital cardiac arrest with refractory ventricular fibrillation
}

Takashi Tagami $^{1,2^{*}}$, Hideo Yasunaga ${ }^{2}$ and Hiroyuki Yokota ${ }^{3}$

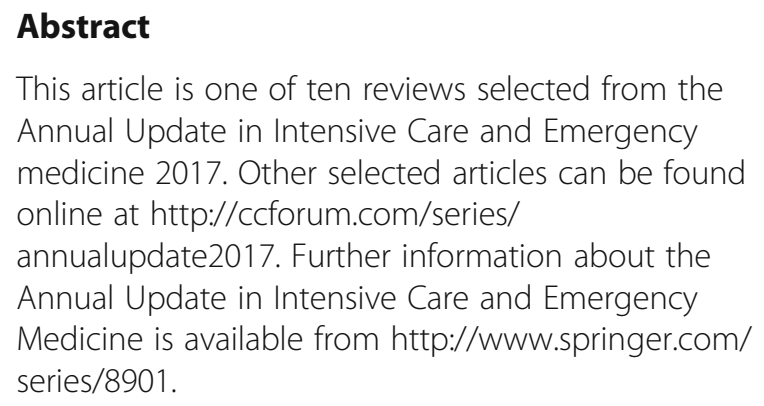

This article is one of ten reviews selected from the Annual Update in Intensive Care and Emergency medicine 2017. Other selected articles can be found online at http://ccforum.com/series/ annualupdate2017. Further information about the Annual Update in Intensive Care and Emergency Medicine is available from http://www.springer.com/ series/8901.

\section{Background}

Out-of-hospital cardiac arrest (OHCA) affects approximately 300,000 people in the United States, 280,000 people in Europe and 110,000 people in Japan each year [1-3]. Among all the presentations of cardiac arrest (asystole, pulseless electrical activity [PEA], ventricular fibrillation [VF], and pulseless ventricular tachycardia $[\mathrm{pVT}]), \mathrm{VF}$ and $\mathrm{pVT}$ are considered the most treatment responsive, but the rate of survival to hospital discharge after OHCA remains markedly low [1-3]. To overcome this time-sensitive and severe condition that has a low survival rate, the "chain of survival" concept was first introduced by Newman [4] in the 1980s as follows: (1) early access to emergency medical care; (2) early cardiopulmonary resuscitation (CPR); (3) early defibrillation; and (4) early advanced cardiac life support. Even after three decades of accumulation of evidence, the 2015 International Liaison Committee on Resuscitation (ILCOR) guidelines still recommend immediate defibrillation with $\mathrm{CPR}$ as the treatment of choice for $\mathrm{VF} / \mathrm{pVT}$

\footnotetext{
* Correspondence: t-tagami@nms.ac.jp

${ }^{1}$ Department of Emergency and Critical Care Medicine, Nippon Medical School, Tama Nagayama Hospital, 2068512 Tama-shi, Tokyo, Japan

${ }^{2}$ Department of Clinical Epidemiology and Health Economics, The University of Tokyo, School of Public Health, Graduate School of Medicine, 1138555 Bunkyo-ku, Tokyo, Japan

Full list of author information is available at the end of the article
}

in OHCA patients and that antiarrhythmic drugs can be used as advanced life support during cardiac arrest in patients with refractory ventricular dysrhythmias $[5,6]$. Refractory $\mathrm{VF} / \mathrm{pVT}$ is generally defined as failure to terminate VF/pVT with one to three stacked shocks [5].

Antiarrhythmic drugs that may be used include amiodarone, lidocaine, and nifekalant ([5, 6]; Table 1). The ILCOR guidelines recommend the use of amiodarone as first-choice treatment for adult patients with refractory $\mathrm{VF} / \mathrm{pVT}$ to improve the rate of return of spontaneous circulation (ROSC) [5, 6]. Lidocaine and nifekalant are recommended as alternatives to amiodarone in the treatment of refractory VF/pVT in adult patients. However, as mentioned in the knowledge gap section of the latest resuscitation guidelines [5], existing evidence is not enough to suggest that amiodarone is superior to lidocaine and/or nifekalant in terms of the critical outcome of survival to discharge. In a trial involving OHCA patients with $\mathrm{VF} / \mathrm{pVT}$, Dorian et al. [7] reported that the rate of survival to admission was significantly higher in the amiodarone group than in the lidocaine group but found no significant difference in the rate of survival to discharge between the two groups. More recently, Kudenchuk et al. [8] reported survival data for patients with OHCA due to initial VF/ $\mathrm{pVT}$ in the prehospital setting and found that survival rates with amiodarone or lidocaine administration were not significantly higher than with placebo.

Thus, the provision of amiodarone, lidocaine, or nifekalant to OHCA patients with refractory VF/pVT is still controversial in clinical practice. In this chapter, we review the recent evidence from randomized trials and observational studies for the efficacy of antiarrhythmic drugs for OHCA patients with refractory VF/pVT.

\section{Characteristics and evidence of the efficacy of antiarrhythmic drugs}

Lidocaine was traditionally used as the drug of choice for the treatment of OHCA in patients with persistent 
Table 1 Classification and recommendations for the three antiarrhythmic drugs

\begin{tabular}{|c|c|c|c|}
\hline & Amiodarone & Lidocaine & Nifekalant \\
\hline Vaughan Williams classification & Class III & Class Ib & Class III \\
\hline $\begin{array}{l}2015 \text { ILCOR treatment } \\
\text { recommendation [5] }\end{array}$ & $\begin{array}{l}\text { Suggest use of amiodarone in adult } \\
\text { patients with refractory VF/pVT to } \\
\text { improve rates of ROSC. }\end{array}$ & $\begin{array}{l}\text { Suggest use of lidocaine as an } \\
\text { alternative to amiodarone in adult } \\
\text { patients with refractory VF/pVT. }\end{array}$ & $\begin{array}{l}\text { Suggest use of nifekalant as an } \\
\text { alternative to amiodarone in adult } \\
\text { patients with refractory } V F / p V T \text {. }\end{array}$ \\
\hline Level of recommendation & $\begin{array}{l}\text { Weak recommendation, } \\
\text { moderate-quality evidence }\end{array}$ & $\begin{array}{l}\text { Weak recommendation, very- } \\
\text { low-quality evidence }\end{array}$ & $\begin{array}{l}\text { Weak recommendation, very-low- } \\
\text { quality evidence }\end{array}$ \\
\hline
\end{tabular}

ILCOR International Liaison Committee on Resuscitation, VF ventricular fibrillation, $p V T$ pulseless ventricular tachycardia

$\mathrm{VF} / \mathrm{pVT}$ until the early 2000s [9]. Lidocaine is classified as a class Ib agent in the Vaughan-Williams classification. Although lidocaine is low-cost and simple to administer, evidence is not enough to suggest its superiority to other antiarrhythmic drugs or placebo in terms of rate of survival to hospital discharge of OHCA patients with persistent VF/ $\mathrm{pVT}$. One retrospective study suggested that lidocaine may improve the success rate of resuscitation [10], contrary to the negative results reported in other studies [11, 12]. After the reports of two landmark studies on amiodarone [7, 13], resuscitation guidelines were revised with preference for amiodarone over lidocaine in the treatment of cardiac arrest patients with persistent VF/pVT $[5,6,9]$.

Amiodarone and nifekalant are both classified as class III antiarrhythmic agents (potassium channel blockers) in the Vaughan-Williams classification [14, 15]. However, these two class III antiarrhythmic drugs have different pharmacological characteristics. Amiodarone has several effects on ion channels, receptors, and sympathetic activity, in addition to blocking the rapid components of delayed rectifier potassium currents, such as sodium and calcium channel-blocking effects, and $\alpha$ - and $\beta$-receptor blocking actions. In other words, amiodarone has negative inotropic and vasodilatory effects, which may negatively affect hemodynamic status after ROSC in OHCA patients. On the other hand, nifekalant is a pure potassium channel blocker that specifically blocks the rapid component of delayed rectifier potassium currents without blocking sodium or calcium channels [16]. Laboratory studies suggest that nifekalant has no negative inotropic effects and no effect on cardiac conduction and hemodynamic status, whereas amiodarone has these effects via its $\beta$-blocking action $[17,18]$. Although some animal model studies suggested that amiodarone did not contribute to the decrease in defibrillation threshold, nifekalant was found to decrease the defibrillation threshold of ventricular fibrillation [19-21]. Therefore, nifekalant may have advantages over amiodarone for the treatment of refractory $\mathrm{VF} / \mathrm{pVT}$ via defibrillation and post-resuscitation hemodynamic management from a pharmacological point of view. However, data from clinical studies have provided limited robust results $[22,23]$.

Only a few studies have compared the efficacy of amiodarone with that of nifekalant for the treatment of refractory VF/pVT [22-25]. Amino et al. [22] reported no significant differences in the success rate of defibrillation and rate of survival to discharge between nifekalant and amiodarone in OHCA patients with refractory VF/ pVT. However, they reported that the interval between antiarrhythmic drug administration and defibrillation success in the amiodarone group was significantly longer than that in the nifekalant group [22]. This finding was also reported in a retrospective study by Harayama et al. [23], who found that nifekalant achieved faster ROSC after refractory VF/pVT than did amiodarone. More recently, Amino et al. [25] evaluated the results of a large multicenter prospective study (SOS-KANTO 2012 [26]). These investigators retrospectively investigated nifekalant potency and differential effects of two initial amiodarone doses (150 or $300 \mathrm{mg}$ ) as compared with lidocaine doses in the Japanese population. The odds ratios (ORs) for survival to admission were significantly higher in the 150-mg nifekalant or amiodarone group than in the lidocaine group. The authors also reported that 24-hour survival was significantly higher in the nifekalant, 150-mg amiodarone, or 300-mg amiodarone groups than in the lidocaine group. Amino et al. [27] published another post hoc analysis from the SOSKANTO 2012 study. They evaluated the effect of administration of antiarrhythmic drugs (defined as any one among or a combination of lidocaine, nifekalant, and amiodarone) during CPR on 1-month outcome by using propensity score analyses. Logistic regression with propensity scoring demonstrated an OR of 1.92 for 1-month survival in the antiarrhythmic drug group $(p=0.01)$ and 1.44 for favorable neurological outcome at 1 month $(p=0.26)$.

\section{The ROC-ALPS study}

Recently, Resuscitation Outcomes Consortium (ROC) investigators published the results of the Amiodarone, Lidocaine or Placebo Study (ALPS), a randomized double-blind trial [8]. Amiodarone, lidocaine, or placebo was administered by paramedics in the prehospital setting for OHCA patients with refractory $\mathrm{VF} / \mathrm{pVT}$ in 10 North American sites. The investigators evaluated 3,026 patients, of whom 974 were assigned to the amiodarone group, 993 to the lidocaine group, and 1,059 to the placebo group. Of these patients, $24.4 \%, 23.7 \%$, and $21.0 \%$, 
respectively, survived to hospital discharge (primary outcome). The difference in survival rate was 3.2 percentage points for amiodarone compared with placebo (95\% confidence interval $[\mathrm{CI}]-0.4$ to $7.0 ; p=0.08), 2.6$ percentage points for lidocaine compared with placebo $(95 \% \mathrm{CI}-1.0$ to $6.3 ; p=0.16$ ), and 0.7 percentage points for amiodarone compared with lidocaine ( $95 \% \mathrm{CI}-3.2$ to $4.7 ; p=0.70)$.

The survival rates with favorable neurological status (secondary outcome) were similar in the amiodarone group (182 patients [18.8\%]), lidocaine group (172 [17.5\%]), and placebo group (175 [16.6\%]). The risk difference for the secondary outcome was 2.2 percentage points for amiodarone compared with placebo ( $95 \% \mathrm{CI}-1.1$ to $5.6 ; p=0.19$ ), 0.9 percentage points for lidocaine compared with placebo (95\% CI -2.4 to $4.2 ; p=0.59$ ), and 1.3 percentage points for amiodarone compared with lidocaine ( $95 \% \mathrm{CI}-2.1$ to $4.8 ; p=0.44$ ).

Kudenchuk et al. [8] concluded the ROC-ALPS study as follows: neither amiodarone nor lidocaine resulted in a survival rate or favorable neurological outcome that was significantly better than that achieved with placebo among patients with OHCA due to initial shock-refractory VF/ pVT. However, the study also provided insightful data to the field of resuscitation. The authors reported that among 1,934 patients with bystander-witnessed arrest, the survival rate was higher with amiodarone $(27.7 \%)$ or lidocaine administration (27.8\%) than with placebo (22.7\%). This absolute risk difference was significant for amiodarone compared with placebo (5.0 percentage points; $95 \% \mathrm{CI}$ $0.3-9.7 ; p=0.04$ ) and for lidocaine compared with placebo (5.2 percentage points; $95 \%$ CI $0.5-9.9, p=0.03$ ). The survival rate was also higher among amiodarone recipients than among placebo recipients with emergency medical services (EMS)-witnessed arrest, with a risk difference of 21.9 percentage points (95\% CI $5.8-38.0 ; p=0.01$ ). Considering that OHCA with refractory $\mathrm{VF} / \mathrm{pVT}$ is a timesensitive condition with a low survival rate, determining whether OHCA was witnessed or not is a reasonable method of performing subanalyses. The authors also reported that among 839 patients with unwitnessed OHCA, survival did not differ significantly between trial groups. These data may indicate that both amiodarone and lidocaine therapies may be effective for shock-refractory bystander-witnessed OHCA but may be useless in unwitnessed OHCA.

\section{Analyses of data from a Japanese nationwide database: comparisons between amiodarone and nifekalant and between amiodarone and lidocaine}

Nifekalant was developed and approved for clinical use in Japan in 1999. Thus, nifekalant and lidocaine were the traditionally used drugs of choice for $\mathrm{VF} / \mathrm{pVT}$ until the Japanese Ministry of Health, Labour and Welfare approved the use of intravenous amiodarone in June 2007. Thus, Japan is the only country in which amiodarone, nifekalant, and lidocaine are all approved for clinical use. We therefore decided to analyze the effectiveness of antiarrhythmic drugs by using real-world clinical data from cardiogenic OHCA patients with refractory VF/ pVT on hospital arrival across the country.

We retrospectively evaluated data from the Japanese Diagnosis Procedure Combination (DPC) database, which is a nationwide in-hospital patient administrative database [28-30]. The DPC database includes administrative claims and discharge abstract data for all patients (including OHCA patients who die in the emergency room) discharged from more than 1,000 participating hospitals, covering all 82 academic hospitals and more than $90 \%$ of all tertiary-care emergency hospitals in Japan. The database includes the following information for each patient, recorded using a uniform data submission form: age, sex, medical procedures (e. g., defibrillation, therapeutic hypothermia, and percutaneous coronary angiogram/ intervention), daily records of all drugs administered and devices used (e. g., amiodarone, nifekalant, lidocaine, extracorporeal membrane oxygenation [ECMO], and intra-aortic balloon pumping [IABP]), length of hospital stay, and discharge status (home, transfer to another hospital, death in the emergency room, or death after admission) [28-30].

We compared the rates of survival to discharge among cardiogenic OHCA patients with persistent VF/pVT on hospital arrival following treatment with either amiodarone or nifekalant [28], and amiodarone or lidocaine [29].

In the study that compared amiodarone and nifekalant [28], we identified 2,961 patients with cardiogenic OHCA who had VF/pVT on hospital arrival between July 2007 and March 2013. Patients were categorized into amiodarone $(\mathrm{n}=2,353)$ and nifekalant groups $(n=608)$, from which 525 propensity score-matched pairs were generated. We found a significant difference in admission rate between the nifekalant and amiodarone groups in the propensity score-matched groups (75.6 vs. 69.3\%; difference 6.3\%; 95\% CI 0.9-11.7). An analysis that used the in-hospital administration rate of nifekalant/amiodarone as an instrumental variable found that receiving nifekalant was associated with an improved admission rate $(22.2 \%$; $95 \%$ CI 11.9-32.4). However, we found no significant difference in in-hospital mortality between the nifekalant and amiodarone groups ( 81.5 vs. $82.1 \%$; difference $-0.6 \%$; $95 \%$ CI -5.2 to 4.1). Instrumental variable analysis showed that receiving nifekalant was not associated with reduced in-hospital mortality $(6.2 \%, 95 \% \mathrm{CI}-2.4$ to 14.8$)$. We concluded that although nifekalant may potentially improve hospital admission rates compared with amiodarone for these patients, no significant association with in-hospital mortality was found between nifekalant 
and amiodarone for cardiogenic OHCA patients with VF/ pVT on hospital arrival.

In the study that compared amiodarone and lidocaine [29], we identified 3,951 patients from 795 hospitals who experienced cardiogenic OHCA and had refractory ventricular fibrillation on hospital arrival. The patients were categorized into amiodarone $(n=1,743)$ and lidocaine $(n=2,208)$ groups, from which 801 propensity scorematched pairs were generated. No significant difference in the rate of survival to hospital discharge was found between the amiodarone and lidocaine groups (15.2 vs. $17.1 \%$; difference $-1.9 \%$; $95 \% \mathrm{CI}-5.5$ to 1.7 ) in the propensity score-matched analyses. Thus, we concluded that the amiodarone and lidocaine groups had no significant difference in the rate of survival to hospital discharge.

As compared with those reported in the ROC-ALPS study, the survival rates of our two nationwide observational studies were lower (approximately $17-18 \%$ in our studies $[28,29]$ vs. $24 \%$ in the ROC-ALPS study [8]). These differences may be attributed to the differences in the EMS of the countries in which the studies were conducted. The ROC-ALPS study [8] was conducted in North America, whereas we analyzed Japanese data. In the ROCALPS study [8], patients were randomized at the scene by the responding paramedics. This is in contrast to our study, in which antiarrhythmic drugs were provided after hospital arrival because paramedics are not allowed to administer any antiarrhythmic drugs in prehospital settings in Japan. Thus, the times from cardiac arrest to first administration of the antiarrhythmic drug were much longer in our studies $[28,29]$ than in the ROC-ALPS study [8].

\section{Recent systematic review and meta-analyses}

Huang et al. [31] reviewed 10 randomized controlled trials and 7 observational trials in 2013. They found that amiodarone (relative risk [RR] 0.82; 95\% CI 0.54-1.24), lidocaine (RR 2.26; 95\% CI 0.93-5.52) and nifekalant therapies did not improve survival to hospital discharge compared with placebo, but amiodarone, lidocaine, and nifekalant therapies were beneficial to initial resuscitation, as assessed based on the rate of ROSC and survival to hospital admission, with amiodarone being superior to lidocaine (RR 1.28; 95\% CI 0.57-2.86) and nifekalant (RR 0.50; 95\% CI 0.19-1.31).

Amino et al. reported a systematic review of the use of intravenous amiodarone and nifekalant in 2014 [32]. They reviewed 9 articles, including those written in the Japanese language. They found that amiodarone and nifekalant therapies were equally effective in preventing electrical storm (67 vs. 67\%). The defibrillation effect on CPR was also equal in the two groups (60 vs. $54 \%$ ). More cases of hypotension and bradycardia were recorded as adverse effects in the amiodarone group (9.5 and 5.3\%) than in the nifekalant group [32].
After publication of the results of the ROC-ALPS study, two systematic reviews with meta-analyses were published in 2016 [33, 34]. Laina et al. [34] reported that amiodarone therapy significantly improved survival to hospital admission (OR 1.402; 95\% CI 1.068-1.840; $Z=$ 2.43; $p=0.015$ ), but neither survival to hospital discharge (RR 0.850; 95\% CI 0.631-1.144; $Z=1.07 ; p=0.284$ ) nor neurological outcome (OR 1.114; $95 \%$ CI $0.923-1.345 ; Z=$ $1.12 ; p=0.475$ ) were significantly improved by amiodarone therapy compared with placebo or nifekalant therapy. Sanfilippo et al. [33] reported another review regarding this issue. They reported that amiodarone was as beneficial as lidocaine for survival to hospital admission (primary analysis OR 1.02; 95\% CI $0.86-1.23 ; p=0.40$ ) and discharge (primary analysis OR $1.06 ; 95 \%$ CI $0.87-1.30 ; p=0.56$; secondary analysis OR $1.04 ; 95 \%$ CI $0.86-1.27 ; p=0.67$ ). Compared with placebo, survival to hospital admission was higher with both amiodarone (primary analysis OR 1.32; 95\% CI 1.12-1.54; $p<0.0001$; secondary analysis OR 1.25 ; 95\% CI 1.07-1.45; $p<0.005$ ) and lidocaine (secondary analysis only OR $1.34 ; 95 \%$ CI $1.14-1.58 ; p=0.0005$ ) therapies. With regard to hospital discharge, no significant differences were observed between placebo and amiodarone (primary outcome OR $1.19 ; 95 \%$ CI $0.98-1.44 ; p=0.08$; secondary outcome OR 1.11; 95\% CI 0.92-1.33; $p=0.28$ ) or lidocaine (secondary outcome only OR $1.19 ; 95 \%$ CI $0.97-1.45$; $p=0.10)$ therapy.

These recent four systematic reviews with meta-analyses suggested that amiodarone, lidocaine and nifekalant therapies equally improved survival to hospital admission as compared with placebo. However, none of these antiarrhythmic drugs improved long-term outcomes.

\section{Is earlier provision of antiarrhythmic drugs better?}

After publication of the results of the ROC-ALPS study, several corresponding editorials and comments were published in response [35-38]. As pointed out by several comments, the key finding of the ROC-ALPS study was that both amiodarone and lidocaine therapies may be effective for shock-refractory $\mathrm{VF} / \mathrm{pVT}$ in bystander-witnessed arrest but might be useless in the later phase of resuscitation $[35,37]$. The first dose of the trial drugs was given a mean $( \pm \mathrm{SD})$ of $19.3 \pm 7.4 \mathrm{~min}$ after the initial call to EMS and after a median of three shocks in the overall population of the ROC-ALPS study [8]. Kudenchuk et al. speculated that such delays may attenuate the effectiveness of antiarrhythmic interventions as patients progress to the metabolic phase of OHCA when cellular injury and physiological derangements may be irreversible despite restored circulation [39]. The potential benefit of treatment could have been underestimated among patients with unwitnessed OHCA. Patients with witnessed OHCA who received earlier initiation of resuscitation and a shorter interval to antiarrhythmic drug 
administration (12 min) had significantly higher rates of survival to hospital discharge by about $5 \%$.

\section{Conclusion}

Recent guidelines on resuscitation recommend immediate defibrillation with CPR as the treatment of choice for OHCA patients with VF/pVT and that antiarrhythmic drugs (including amiodarone, lidocaine and nifekalant) can be used as advanced life support during cardiac arrest in patients with refractory ventricular dysrhythmias. Recent systematic reviews and meta-analyses suggest that amiodarone, lidocaine, and nifekalant therapies equally improve survival to hospital admission compared with placebo. However, none of these antiarrhythmic drugs improved long-term outcomes. The landmark study, ROC-ALPS, concluded that compared with placebo, neither amiodarone nor lidocaine therapy resulted in a significantly higher rate of survival or more favorable neurological outcome among patients with OHCA due to refractory $\mathrm{VF} / \mathrm{pVT}$. However, the authors also reported that among patients with bystander-witnessed cardiac arrest, the survival rate was higher with amiodarone or lidocaine than with placebo. Although further studies are required to confirm this speculation, earlier administration of antiarrhythmic drugs may result in better outcomes.

\section{Funding}

This work was supported by Grants for Research on Policy Planning and Evaluation from the Ministry of Health, Labour and Welfare, Japan (Grant Numbers: H28-Policy-Designated-009 to Prof. Yasunaga; H27-Policy-Strategy-011 to Prof. Yasunaga), and a Grant-in-Aid for Scientific Research, Japan (Grant Number: KAKENHI-15H05685 to Dr. Tagami). The funders had no role in the execution of this study or interpretation of the results. The publication costs were funded by Grant-in-Aid for Scientific Research, Japan (Grant Number: KAKENHI-15H05685 to Dr. Tagami).

\section{Availability of data and materials}

Not applicable.

\section{Authors' contributions}

$\Pi \mathrm{h}$ had full access to all the study data and take responsibility for the integrity of the data and the accuracy of the data analysis, and drafted the manuscript. $\mathrm{HY}$ and $\mathrm{HY}$ participated in the study design, analysis and interpretation of the data, and helped to drafted the manuscript. All authors have critically revised the manuscript and gave approval for the final manuscript.

\section{Competing interests}

The authors declare that they have no competing interests.

\section{Consent for publication}

Not applicable.

Ethics approval and consent to participate Not applicable.

\section{Author details}

'Department of Emergency and Critical Care Medicine, Nippon Medical School, Tama Nagayama Hospital, 2068512 Tama-shi, Tokyo, Japan. ${ }^{2}$ Department of Clinical Epidemiology and Health Economics, The University of Tokyo, School of Public Health, Graduate School of Medicine, 1138555 Bunkyo-ku, Tokyo, Japan. ${ }^{3}$ Department of Emergency and Critical Care Medicine, Nippon Medical School, 1138603 Bunkyo-ku, Tokyo, Japan.
Published online: 21 March 2017

References

1. Atwood C, Eisenberg MS, Herlitz J, Rea TD. Incidence of EMS-treated out-ofhospital cardiac arrest in Europe. Resuscitation. 2005;67:75-80.

2. Kitamura T, Iwami T, Kawamura T, et al. Nationwide improvements in survival from out-of-hospital cardiac arrest in Japan. Circulation. 2012;126:2834-43.

3. Nichol G, Thomas E, Callaway CW, et al. Regional variation in out-of-hospital cardiac arrest incidence and outcome. JAMA. 2008:300:1423-31.

4. Newman M. The chain of survival concept takes hold. JEMS. 1989;14:11-3.

5. Callaway CW, Soar J, Aibiki M, et al. Part 4: Advanced Life Support: 2015 International Consensus on Cardiopulmonary Resuscitation and Emergency Cardiovascular Care Science With Treatment Recommendations. Circulation. 2015;132:84-S145

6. Soar J, Nolan JP, Bottiger BW, et al. European Resuscitation Council Guidelines for Resuscitation 2015: Section 3. Adult advanced life support. Resuscitation. 2015;95:100-47.

7. Dorian P, Cass D, Schwartz B, Cooper R, Gelaznikas R, Barr A. Amiodarone as compared with lidocaine for shock-resistant ventricular fibrillation. $N$ Engl J Med. 2002:346:884-90.

8. Kudenchuk PJ, Brown SP, Daya M, et al. Amiodarone, lidocaine, or placebo in out-of-hospital cardiac arrest. N Engl J Med. 2016;374:1711-22.

9. The American Heart Association in collaboration with the International Liaison Committee on Resuscitation. Guidelines 2000 for Cardiopulmonary Resuscitation and Emergency Cardiovascular Care. Part 6: advanced cardiovascular life support: section 5: pharmacology l: agents for arrhythmias. Circulation. 2000;102:1112-128.

10. Herlitz J, Ekstrom L, Wennerblom B, et al. Lidocaine in out-of-hospital ventricular fibrillation. Does it improve survival? Resuscitation. 1997;33:199-205.

11. Harrison EE. Lidocaine in prehospital countershock refractory ventricular fibrillation. Ann Emerg Med. 1981;10:420-3.

12. Weaver WD, Fahrenbruch CE, Johnson DD, Hallstrom AP, Cobb LA, Copass MK. Effect of epinephrine and lidocaine therapy on outcome after cardiac arrest due to ventricular fibrillation. Circulation. 1990;82:2027-34.

13. Kudenchuk PJ, Cobb LA, Copass MK, et al. Amiodarone for resuscitation after out-of-hospital cardiac arrest due to ventricular fibrillation. N Engl J Med. 1999:341:871-8.

14. Singh BN, Vaughan Williams EM. The effect of amiodarone, a new antianginal drug, on cardiac muscle. Br J Pharmacol. 1970;39:657-67.

15. Pantazopoulos IN, Troupis GT, Pantazopoulos CN, Xanthos TT. Nifekalant in the treatment of life-threatening ventricular tachyarrhythmias. World J Cardiol. 2011;3:169-76.

16. Nakaya H, Tohse N, Takeda Y, Kanno M. Effects of MS-551, a new class III antiarrhythmic drug, on action potential and membrane currents in rabbit ventricular myocytes. Br J Pharmacol. 1993;109:157-63.

17. Kondoh $\mathrm{K}$, Hashimoto $\mathrm{H}$, Nishiyama $\mathrm{H}$, et al. Effects of MS-551, a new class III antiarrhythmic drug, on programmed stimulation-induced ventricular arrhythmias, electrophysiology, and hemodynamics in a canine myocardial infarction model. J Cardiovasc Pharmacol. 1994;23:674-80.

18. Sen L, Cui G, Sakaguchi Y, Singh BN. Electrophysiological effects of MS-551, a new class III agent: comparison with dl-sotalol in dogs. J Pharmacol Exp Ther. 1998;285:687-94.

19. Murakawa Y, Yamashita T, Kanese Y, Omata M. Can a class III antiarrhythmic drug improve electrical defibrillation efficacy during ventricular fibrillation? J Am Coll Cardiol. 1997:29:688-92.

20. Tsagalou EP, Anastasiou-Nana MI, Charitos CE, et al. Time course of fibrillation and defibrillation thresholds after an intravenous bolus of amiodarone - an experimental study. Resuscitation. 2004;61:83-9.

21. Huang J, Skinner JL, Rogers JM, Smith WM, Holman WL, Ideker RE. The effects of acute and chronic amiodarone on activation patterns and defibrillation threshold during ventricular fibrillation in dogs. J Am Coll Cardiol. 2002;40:375-83.

22. Amino M, Yoshioka $\mathrm{K}$, Opthof $\mathrm{T}$, et al. Comparative study of nifekalant versus amiodarone for shock-resistant ventricular fibrillation in out-of-hospital cardiopulmonary arrest patients. J Cardiovasc Pharmacol. 2010;55:391-8.

23. Harayama N, Nihei S, Nagata K, et al. Comparison of nifekalant and amiodarone for resuscitation of out-of-hospital cardiopulmonary arrest resulting from shock-resistant ventricular fibrillation. J Anesth. 2014;28:587-92

24. Ji XF, Li CS, Wang S, Yang L, Cong LH. Comparison of the efficacy of nifekalant and amiodarone in a porcine model of cardiac arrest. Resuscitation. 2010;81:1031-6. 
25. Amino M, Inokuchi S, Nagao K, et al. Nifekalant hydrochloride and amiodarone hydrochloride result in similar improvements for 24-hour survival in cardiopulmonary arrest patients: The SOS-KANTO 2012 Study. J Cardiovasc Pharmacol. 2015;66:600-9.

26. SOS-KANTO 2012 Study group. Changes in pre- and in-hospital management and outcomes for out-of-hospital cardiac arrest between 2002 and 2012 in Kanto, Japan: the SOS-KANTO 2012 Study. Acute Med Surg. 2015;2:225-33.

27. Amino M, Inokuchi S, Yoshioka $K$, et al. Does antiarrhythmic drug during cardiopulmonary resuscitation improve the one-month survival: The SOS-KANTO 2012 Study. J Cardiovasc Pharmacol. 2016;68:58-66.

28. Tagami T, Matsui H, Ishinokami S, et al. Amiodarone or nifekalant upon hospital arrival for refractory ventricular fibrillation after out-of-hospital cardiac arrest. Resuscitation. 2016;109:127-32.

29. Tagami T, Matsui H, Tanaka C, et al. Amiodarone compared with lidocaine for out-of-hospital cardiac arrest with refractory ventricular fibrillation on hospital arrival: a nationwide database study. Cardiovasc Drugs Ther. 2016;30:485-91.

30. Tagami T, Matsui H, Fushimi K, Yasunaga H. Changes in therapeutic hypothermia and coronary intervention provision and in-hospital mortality of patients with out-of-hospital cardiac arrest: a nationwide database study. Crit Care Med. 2016;44:488-95.

31. Huang $Y, H e ~ Q$, Yang M, Zhan L. Antiarrhythmia drugs for cardiac arrest: a systemic review and meta-analysis. Crit Care. 2013;17:R173.

32. Amino M, Yoshioka K, Kanda S, et al. Systematic review of the use of intravenous amiodarone and nifekalant for cardiopulmonary resuscitation in Japan. J Arrhyth. 2014;30:180-5

33. Sanfilippo F, Corredor C, Santonocito C, et al. Amiodarone or lidocaine for cardiac arrest: A systematic review and meta-analysis. Resuscitation. 2016;107:31-7.

34. Laina A, Karlis G, Liakos A, et al. Amiodarone and cardiac arrest: Systematic review and meta-analysis. Int J Cardiol. 2016;221:780-8.

35. Sugiyama K, Kashiura M, Hamabe Y. Amiodarone and lidocaine for shock refractory ventricular fibrillation or ventricular tachycardia in out-of-hospital cardiac arrest: are they really effective? J Thorac Dis. 2016;8:E791-3.

36. Ho AF, Ong ME. Antiarrhythmic drugs in out-of-hospital cardiac arrest-what does the Amiodarone, Lidocaine, or Placebo Study tell us? J Thorac Dis. 2016;8:E604-6

37. Joglar JA, Page RL. Out-of-hospital cardiac arrest - are drugs ever the answer? N Engl J Med. 2016;374:1781-2.

38. Kudenchuk PJ, Daya M, Dorian P, Resuscitation Outcomes Consortium I. Amiodarone, lidocaine, or placebo in out-of-hospital cardiac arrest. N Engl J Med. 2016;375:802-3.

39. Weisfeldt ML, Becker LB. Resuscitation after cardiac arrest: a 3-phase timesensitive model. JAMA. 2002;288:3035-8. 